\title{
Laser induced surface structures on gold-coated polymers: influence of morphology on Surface-Enhanced Raman Scattering enhancement
}

\author{
Esther Rebollar ${ }^{a}$, Margarita Hernández ${ }^{b}$, Mikel Sanz $^{a}$, Susana Pérez ${ }^{a}$, Tiberio A. Ezquerra ${ }^{b}$, Marta \\ Castillejo $^{a}$ \\ a Instituto de Química Física Rocasolano, IQFR-CSIC, Serrano 119, 28006 Madrid, Spain. \\ ${ }^{\mathrm{b}}$ Instituto de Estructura de la Materia, IEM-CSIC, Serrano 121, 28006 Madrid, Spain. \\ Correspondence to: Esther Rebollar (E-mail: e.rebollar@csic.es)
}

\begin{abstract}
The fabrication of highly sensitive and reproducible substrates for Surface Enhanced Raman Scattering (SERS) remains a challenging scientific and technological issue. In this work laser induced periodic surface structures are generated on poly(trimethylen terephthalate) films upon laser irradiation with the linearly polarized beams of a Nd:YAG laser ( $4^{\text {th }}$ harmonic, $266 \mathrm{~nm}$ ), an ArF excimer laser $(193 \mathrm{~nm})$ and a Titanium:sapphire laser $(795 \mathrm{~nm})$, resulting in periods close to the laser wavelength when irradiating at normal incidence, and larger periods for different angles of incidence. Additional irradiation with a circularly polarized beam at $266 \mathrm{~nm}$ produces superficial circular structures. The nanostructured polymers are coated with a nanoparticle assembled gold layer by pulsed laser deposition at $213 \mathrm{~nm}$. The capabilities of these substrates for SERS are evaluated using benzenethiol as a test molecule and different degrees of Raman signal enhancement are observed depending on the nanostructure type. The highest enhancement factor is obtained by for nanostructured substrates with the highest values of period, depth and roughness.
\end{abstract}

KEYWORDS Laser induced periodic surface structures, polymer films, pulsed laser deposition, gold coatings, surface enhanced Raman scattering

\section{INTRODUCTION}

Surface plasmons induced by an incident laser electromagnetic field on noble metal surfaces constitute the basis of Surface-Enhanced Raman Scattering (SERS) ${ }^{1-4}$. The intensification of the Raman signal leads to outstanding sensitivities that can reach the limit of single molecule detection ${ }^{5}$ and enable SERS substrates for use in non-invasive biological assays ${ }^{6}$, biosensing ${ }^{7}$ 
or environmental analysis ${ }^{8}$. Soon after the discovery of SERS it was recognized that the substrate morphology plays a crucial role to the Raman intensity enhancement. In particular, for corrugated surfaces made of nanoparticle assemblies, the enhancement of spectral features is highly sensitive to size, shape and degree of aggregation of nanoparticles ${ }^{9-11}$. Thus, controlling these parameters is a major issue when pointing at the selective detection of ultralow molecular concentrations. The development of SERS substrates with good enough sensitivity, reproducibility, robustness and stability has become an active field of research ${ }^{12}$ and several different methods have been employed to fabricate SERS-active substrates. These include immobilization of colloidal nanoparticles on solid surfaces ${ }^{13}$, lithographic techniques ${ }^{14-}$ ${ }^{17}$, and other procedures of preparation of nanostructured metallic surfaces ${ }^{18-27}$, including laser induced periodic surface structuring (LIPSS) ${ }^{26,28}$.

Complex nanostructured substrates consisting of polymers coated with a metal layer are applied in different fields as packaging materials, filtering elements, electronic, photovoltaic, optical and mechanical devices, flexible displays and nanosensing and nanomedical components ${ }^{29-34}$. With the combination of such different materials one can merge the excellent properties of polymers, in terms of mechanical flexibility, light weight, enhanced durability and low cost, with particular metallic properties like electrical conductivity and plasmonic properties. In fact, latest years have witnessed growing interest in the fabrication of flexible plasmonic substrates ${ }^{35}$ for non-invasive sensing. The research on flexible plasmonics is quickly moving toward the fabrication of low-cost devices for daily-life applications and recent studies have reported the fabrication of flexible SERS substrates made of membranes of natural rubber 
and polydimethylsiloxane containing gold nanoparticles ${ }^{36,37}$, of silver nanoparticles embedded in polycarbonate plastic films ${ }^{38}$ and of hard thermoplastic polymers coated by thin gold or silver layers by sputtering and electroless deposition ${ }^{39}$.

Recently some of us have reported on enhancement of the Raman signal of benzenethiol (BT) induced by a nanoparticle-assembled gold layer on flat poly(trimethylene terephthalate) (PTT)

films used as substrates ${ }^{26}$. This enhancement, up to eight orders of magnitude, is further increased, by a factor of 10, when the polymer films are endowed with LIPSS with a period of about $250 \mathrm{~nm}$. In order to investigate the full potential of the proposed substrate architectures and to further increase the Raman signal enhancement, we have undertaken in this work the fabrication and characterization of SERS substrates based on a range of gold-coated superficially nanostructured polymer films. To understand how the Raman signal depends on the shape and dimensions of the underlying polymer nanostructures we have generated LIPSS gratings by laser radiation at 193, 266 and $795 \mathrm{~nm}$ and circular superficial structures with a circularly polarized laser beam at $266 \mathrm{~nm}$. The nanostructured polymer films were then coated with gold by PLD, using the fifth harmonic of a Nd:YAG laser at $213 \mathrm{~nm}$, and characterized by Atomic Force Microscopy (AFM). Testing of the SERS performance was done using BT as a probe molecule. The results clearly show the influence of the nanostructure dimensions on the enhancement factor.

\section{EXPERIMENTAL}

\section{Substrates nanostructuring and characterization}


Polymer thin films of poly (trimethylene terephthalate) (PTT) ${ }^{40}$ with molecular weight $M_{n}=31300 \mathrm{~g} \cdot \mathrm{mol}^{-1}$ and a polydispersity $M_{w} / M_{n}=2.2$, were prepared by spin coating on silicon wafers (100) (Wafer World Inc.). The wafers were previously cleaned with acetone and isopropanol, and dried with a nitrogen jet. PTT was solved in trifluoroacetic acid (Sigma-Aldrich, reagent grade $\geq 98 \%$ ) in a concentration of $20 \mathrm{gL}^{-1}$ and a fixed amount of $0.1 \mathrm{~mL}$ of polymer solution was instantly dropped by a syringe on a rectangular $\left(2 \times 2.5 \mathrm{~cm}^{2}\right)$ silicon substrate placed in the centre of a rotating horizontal plate. A rotation speed of $2380 \mathrm{rpm}$ was kept during $30 \mathrm{~s}$. Under these conditions, spin-coated polymer films with a thickness of ca. $150 \mathrm{~nm}$ and smooth surface, with mean roughness values $\left(R_{a}\right)$ under $1 \mathrm{~nm}$, as measured by AFM, are typically obtained $^{41,42}$.

For the LIPSS fabrication laser irradiation of the thin polymer films was carried out in ambient air using three different laser systems. The first system is constituted by the linearly polarized beam of the fourth harmonic of a Q-switched Nd:YAG laser (Quantel Brilliant B, $266 \mathrm{~nm}$, pulse duration $6 \mathrm{~ns}$ ) at a repetition rate of $10 \mathrm{~Hz}$. In this case, the polymer films were irradiated by selecting the central part of the beam with an iris of $4.5 \mathrm{~mm}$ diameter situated in front of the sample. The laser pulse energy was measured with a joulemeter and fluences of $7 \mathrm{~mJ} \cdot \mathrm{cm}^{-2}$ were used for irradiation ${ }^{41}$. Larger irradiated areas, of around $4.5 \times 10 \mathrm{~mm}^{2}$, were generated by scanning the sample in the transverse direction at a speed of $75 \mu \mathrm{m} \cdot \mathrm{s}^{-1}$ that ensured the delivery of the optimal amount of pulses for LIPSS formation (ca. 1200). Polymer films were also irradiated at an angle of incidence of $45^{\circ}$ to produce structures with a period larger than the 
laser wavelength. Additionally, samples were irradiated with circularly polarized laser by inserting a quarter-wave plate on the $266 \mathrm{~nm}$ beam path.

The second system consists of an ArF laser (MPB AQX-150) that operates at a wavelength of $193 \mathrm{~nm}$, with pulse duration $10 \mathrm{~ns}$ and repetition rate of $4 \mathrm{~Hz}$. The laser output was linearly polarized with a $\mathrm{MgF}_{2}$ Rochon prism (Bernhard Halle PLM 10) and presented a rectangular near field beam intensity distribution. For homogeneous illumination of the samples we used an aperture of $2 \times 4 \mathrm{~mm}^{2}$ and a spherical lens of $15 \mathrm{~cm}$ focal length placed $6.5 \mathrm{~cm}$ away from the sample. In this case the samples were irradiated with 600 pulses of $3 \mathrm{~mJ} \cdot \mathrm{cm}^{-2}$.

Finally, we used a third laser source consisting of a Ti:sapphire oscillator (Tsunami, Spectra Physics) and a regenerative amplifier system (Spitfire, Spectra Physics) based on the chirped pulse amplification technique. The system produces $120 \mathrm{fs}$ pulses at $795 \mathrm{~nm}$ with a repetition rate of $1 \mathrm{kHz}$. The pulse energy is finely controlled by a half-wave plate and a linear polarizer and we used neutral density filters for further energy reduction. The average power of the beam was measured with a thermopile detector. The transversal mode is nearly a Gaussian TEM00 with a $10 \mathrm{~mm}$ beam diameter $\left(\right.$ at $\left.1 / \mathrm{e}^{2}\right)$. For irradiation, the pulses were focused on the surface with a biconvex, achromatic spherical lens of $100 \mathrm{~mm}$ focal length. Again, larger processed areas were irradiated by sample scanning at a speed of $150 \mu \mathrm{m} \cdot \mathrm{s}^{-1}$ that ensured delivery of the optimal amount of pulses for LIPSS formation ${ }^{43}$. At this irradiation wavelength we prepared irradiated areas of around $4 \times 4 \mathrm{~mm}^{2}$ with 50000 pulses of $37 \mathrm{~mJ} \cdot \mathrm{cm}^{-2}$.

The nanostructured polymer films were then coated with gold by PLD ${ }^{26}$. The gold targets $(99.99 \%$ purity; Quorum Technologies, Kent, UK) were ablated in a set up previously described ${ }^{26,44}$ and 
based on a stainless-steel vacuum deposition chamber pumped down to $6 \times 10^{-4} \mathrm{~Pa}$ by a turbomolecular pump. Deposits were obtained by ablation with a Q-switched Nd:YAG (Lotis II, LS2147 ) operating at $213 \mathrm{~nm}$ ( $5^{\text {th }}$ harmonic of the fundamental radiation, pulse duration of $15 \mathrm{~ns}$ ), at a repetition rate of $10 \mathrm{~Hz}$. The laser beam was focused by a $25 \mathrm{~cm}$ focal length lens to yield a fluence of $2 \mathrm{~J} \cdot \mathrm{cm}^{-2}$ on the rotating target surface. Gold layers were grown on the polymer samples, kept at room temperature and placed $4 \mathrm{~cm}$ away from the target, by delivering 12000 pulses to the metallic target (deposition time of $20 \mathrm{~min}$ ). For this number of pulses, the thickness and roughness of the gold layer were determined by AFM, resulting in values of $3.3 \pm$ $0.6 \mathrm{~nm}$ and $1.5 \pm 0.1 \mathrm{~nm}$ respectively, values which were found optimal for SERS signal enhancement $^{26}$.

The topography of the polymer films was examined by AFM (Multimode 8, Bruker). Analysis was performed in tapping mode, and images were analyzed with the software Nanoscope Analysis 1.40 .

\section{SERS measurements}

As a liquid analyte we used benzenethiol (BT) (> 98\% purity) which was obtained from Fluka Chemika. Diluted aqueous solutions of this analyte were prepared with Milli-Q water. A solution drop with a volume of ca. $5 \mu \mathrm{L}$ and a concentration of $10^{-4} \mathrm{M}$ was poured onto the prepared SERS substrates and dried in air. Raman spectra were recorded with a micro-Raman spectrometer (Renishaw InVia 0310-02), equipped with a CCD and a diode laser emitting at 785 $\mathrm{nm}$ as the excitation source, operating at a power level of around $2 \mathrm{~mW}$ ( $1 \%$ of the total laser power). The spectra were acquired by collecting scattered light in back scattering geometry, 
using a x100 magnification objective (NA 0.85). Acquisition time and accumulations were $10 \mathrm{~s}$ and 10 respectively. The spectral resolution was set to $2 \mathrm{~cm}^{-1}$ and measurements were performed at four different positions of the sample.

\section{RESULTS AND DISCUSSION}

Polymer films were irradiated under selected laser conditions resulting in different surface structures in terms of shape and size. Table 1 lists the characteristic parameters of the different samples, together with the laser irradiation conditions.

The topography of the polymer substrates as revealed by AFM and the corresponding height images are shown in Figure 1a. The period of the obtained structures is, as expected, of the order of the laser wavelength for irradiation at normal incidence. For irradiation at $266 \mathrm{~nm}$ with an incidence angle of $45^{\circ}$, the period increases according to the well-known expression ${ }^{45}$ $L=\lambda /((n-\sin (\theta))$ were $\lambda$ is the laser wavelength and $n$ the effective refractive index. Circular structures, with an average distance between the structure centres of the order of the laser wavelength, were obtained with circularly polarized laser light. The depth of the superficial structures, listed in Table 1, also depends on the irradiation parameters, ranging from 25 to 110 $\mathrm{nm}$.

The samples were then coated with a thin layer of gold by PLD which showed strong adhesion to the nanostructured polymer films ${ }^{26}$. The morphology of the gold deposits was also characterized by AFM and the corresponding height images and profiles are shown in Figure $1 \mathrm{~b}$. 
It is observed that the nanostructure is preserved in all the cases, without significant changes of both period and height.

For SERS measurements, a drop of BT $10^{-4} \mathrm{M}$ was poured onto the samples. The interaction of BT with the gold surface causes variations in the Raman bands of the analyte with respect to the spectrum in solution ${ }^{26,46,47}$. In particular, a band at $917 \mathrm{~cm}^{-1}$, observed in the spectrum of the liquid (inset Figure 2), and assigned to the in plane bending of the $\mathrm{S}-\mathrm{H}$ bond, is no longer detected in the spectrum of BT acquired on the gold-coated substrate. This effect, together with a band shift from $1092 \mathrm{~cm}^{-1}$ to $1071 \mathrm{~cm}^{-1}$ (inset Figure 2), give supporting evidence of the bonding of the benzenethiolate anion to the gold surface via the sulphur atom ${ }^{46}$. Figure 2 shows SERS spectra of $10^{-4} \mathrm{M}$ BT in the range of interest of $900-1150 \mathrm{~cm}^{-1}$ obtained on the goldcoated polymer substrates. The black line refers to the substrate based on a flat, nonnanostructured polymer film, while the coloured lines refer to laser-nanostructured polymer films fabricated with the different laser conditions listed in Table 1.

As previously reported, coating of flat polymer films by a nanoparticle-assembled gold layer increases the Raman signal up to 8 orders of magnitude ${ }^{26}$ as a result of the coupling of localized surface plasmon in the gold nanoparticles. In fact, while concentrations of BT of the order $10 \mathrm{M}$ on a silicon substrate give a negligible Raman signal, the use as substrates of gold coated polymer film allows detection of $10^{-8} \mathrm{M}$ solution of this analyte ${ }^{26}$. Figure 3 shows in detail the topography of the gold layer coating a flat and a nanostructured polymer film and constituted by gold nanoparticles with sizes of up to a few nanometers. Noble metal nanoparticles obtained by PLD have been previously shown to enhance the Raman signal ${ }^{25,48-50}$ 
and this effect was attributed to the relatively high fraction of "hot spots", localized in the junctions ${ }^{51}$. In our case, the enhancement by 8 orders of magnitude of the Raman signal can be attributed to the large number of hot spots in a highly packed nanoparticle metal layer. Figure 3 shows the top of a gold coated periodic structure created by laser on the polymer film and displays a nanoparticle-assembled morphology and roughness of gold layer seems to be the same both on flat and nanostructured polymer films.

Thus, the additional enhancement shown in Figure 2 is caused by nanostructuring of the polymer film located underneath the gold layer. While we measured an enhancement factor of around 10 in the presence of LIPSS with periods of $250 \mathrm{~nm}$ (green line in Figure 2$)^{26}$, the results here presented clearly show that this factor depends on the size and type of the laser created structures. The largest Raman response is achieved for the substrates prepared by irradiation at $266 \mathrm{~nm}$ at an angle of incidence of $45^{\circ}$ (dark blue line in Figure 2) and by irradiation at $795 \mathrm{~nm}$ (light blue line in Figure 2), where the enhancement factor is 33 and 30 respectively. The presence of circular structures increases the signal by a factor of 19, (pink line in Figure 2), while irradiation at $193 \mathrm{~nm}$ (red line in Figure 2) only yields a factor of 2 improvement of the Raman signal. Table 1 summarizes the stated SERS enhancement factors. Inspection of the results reveals that higher Raman enhancement is obtained for the structures with greater period, depth and roughness. This trend can be clearly visualized in Figure 4 . The enhancement factor of the SERS signal seems to be more related to the roughness and depth of the nanostructures than to their period and we attribute this effect to the larger effective area of interaction between substrate and analyte. It is also observed that circular structures induce a 
higher enhancement of the Raman signal in comparison to linear ones of comparable sizes (prepared upon irradiation with the same laser wavelength, fluence and number of pulses), which is in agreement with the idea of the relevance of roughness and effective area of interaction.

\section{CONCLUSIONS}

Gold-coated nanostructured poly(trimethylene terephthalate) thin films have been tested as SERS substrates. Polymer nanostructures of different morphologies and dimensions were prepared by laser irradiation, which resulted in LIPSS or in circular structures. Coating with gold by pulsed laser deposition has been shown to preserve the relief of the nanostructured polymer films and to ensure good adhesion. These substrates have been evaluated for SERS performance using benzenethiol as model analyte. The increase of SERS signal depends on the nanostructure size and morphology. The highest enhancement factor caused by nanostructuring of the underlying polymer film is $\geqslant 30$ for polymer nanostructures obtained by laser irradiation with the highest values of period, depth and roughness, which is attributed to the larger effective area of interaction of the substrate with the analyte. The reported SERS substrates based on PLD gold-coated nanostructured polymers present a number of advantages due to the reduced amount of precious metal used, to the possibility of efficient control of the layer topography, to their sensitivity and uniformity and to the fact that they can be easily fabricated. Additionally, the use of flexible polymer substrates opens new possibilities in the fields of printed electronics and flexible displays.

\section{ACKNOWLEDGEMENTS}


Funding from Ministry of Economy and Competitiveness (MINECO), Spain (Projects CTQ201343086-P, MAT2011-23455 and MAT2012-33517) and Program Geomateriales 2 (S2013/MIT2914) financed by Comunidad de Madrid and Structural Funds (FSE and FEDER), are gratefully acknowledged. E.R. and S.P. thanks MINECO, Spain for a Ramón y Cajal contract (RYC-201108069) and a FPI fellowship, respectively. We thank Z. Roslaniec and A. Szymczyk for giving us the PTT sample, M. Martín for the use of the ArF laser, and P. Moreno and J.R. Vázquez de Aldana for the use of the Ti:sapphire laser. 


\section{REFERENCES AND NOTES}

1. M. Moskovits Rev. Mod. Phys. 1985, 57, 783-826.

2. R. Aroca Surface enhanced vibrational spectroscopy; Wiley: Hoboken, NJ, 2006.

3. E. C. L. Ru and P. G. Etchegoin Principles of surface-enhanced Raman spectroscopy: and related plasmonic effects; Elsevier: Amsterdam, Boston, 2009.

4. P. L. Stiles, J. A. Dieringer, N. C. Shah and R. P. Van Duyne Ann. Rev. Anal. Chem. 2008, 1, 601-626.

5. K. Kneipp, Y. Wang, H. Kneipp, L. T. Perelman, I. Itzkan, R. R. Dasari and M. S. Feld Phys. Rev. Lett. 1997, 78, 1667-1670.

6. S. Feng, J. Lin, Z. Huang, G. Chen, W. Chen, Y. Wang, R. Chen and H. Zeng Appl. Phys. Lett. 2013, 102, 043702.

7. J. Chen, G. Qin, J. Wang, J. Yu, B. Shen, S. Li, Y. Ren, L. Zuo, W. Shen and B. Das Biosens. Bioelectron. 2013, 44, 191-197.

8. L. Guerrini, J. V. Garcia-Ramos, C. Domingo and S. Sanchez-Cortes Anal. Chem. 2009, 81, 1418-1425.

9. C. J. Orendorff, A. Gole, T. K. Sau and C. J. Murphy Anal. Chem. 2005, 77, 3261-3266.

10. K. Faulds, R. E. Littleford, D. Graham, G. Dent and W. E. Smith Anal. Chem. 2004, 76, 592-598.

11. S. Hong and X. Li Journal of Nanomaterials 2013, 2013, 9.

12. M. Fan, G. F. S. Andrade and A. G. Brolo Anal. Chim. Acta 2011, 693, 7-25.

13. M. R. Barmi, C. Andreou, M. R. Hoonejani, M. Moskovits and C. D. Meinhart Langmuir 2013, 29,

13614-13623.

14. C. L. Haynes, C. R. Yonzon, X. Zhang and R. P. Van Duyne J. Raman Spectrosc. 2005, 36, 471-484.

15. S. Sánchez-Cortés, C. Domingo, J. V. García-Ramos and J. A. Aznárez Langmuir 2001, 17, 1157-1162.

16. S.-J. Huo, Q.-X. Li, Yan, Y. Chen, W.-B. Cai, Q.-J. Xu and M. Osawa J. Phys. Chem. B 2005, 109, 1598515991.

17. K. C. Grabar, R. G. Freeman, M. B. Hommer and M. J. Natan Anal. Chem. 1995, 67, 735-743.

18. Y. Lu, G. L. Liu and L. P. Lee Nano Lett. 2004, 5, 5-9.

19. S. Chan, S. Kwon, T. W. Koo, L. P. Lee and A. A. Berlin Adv. Mater. 2003, 15, 1595-1598.

20. S. Zhu, C. Fan, J. Wang, J. He and E. Liang Appl. Phys. A 2014, 117, 1075-1083.

21. S. Kundu, M. Mandal, S. K. Ghosh and T. Pal J. Colloid Interface Sci. 2004, 272, 134-144.

22. H. H. Wang, C. Y. Liu, S. B. Wu, N. W. Liu, C. Y. Peng, T. H. Chan, C. F. Hsu, J. K. Wang and Y. L. Wang Adv. Mater. 2006, 18, 491-495.

23. Y.-C. Tsai, P.-C. Hsu, Y.-W. Lin and T.-M. Wu Sens. Actuators B 2009, 138, 5-8.

24. C. Tian, Y. Deng, D. Zhao and J. Fang Advanced Optical Materials 2015, 3, 404-411.

25. C. Domingo, V. Resta, S. Sanchez-Cortes, J. V. García-Ramos and J. Gonzalo J. Phys. Chem. C 2007, $111,8149-8152$.

26. E. Rebollar, M. Sanz, S. Perez, M. Hernandez, I. Martin-Fabiani, D. R. Rueda, T. A. Ezquerra, C. Domingo and M. Castillejo Phys. Chem. Chem. Phys. 2012, 14, 15699-15705.

27. N. R. Agarwal, M. Tommasini, E. Fazio, F. Neri, R. C. Ponterio, S. Trusso and P. M. Ossi Appl. Phys. A 2014, 117, 347-351.

28. H.-W. Chang, Y.-C. Tsai, C.-W. Cheng, C.-Y. Lin, Y.-W. Lin and T.-M. Wu J. Colloid Interface Sci. 2011, 360, 305-308.

29. V. Kotál, V. Švorčík, P. Slepička, P. Sajdl, O. Bláhová, P. Šutta and V. Hnatowicz Plasma Process. Polym. 2007, 4, 69-76.

30. L. Frisk and E. Ristolainen Microelectron. Rel. 2005, 45, 583-588.

31. D. S. Chang Yao, Thomas J Webster Int. J. Nanomedicine 2007, 2, 487-492. 
32. V. Resta, A. Caricato, A. Loiudice, A. Rizzo, G. Gigli, A. Taurino, M. Catalano and M. Martino J. Nanopart. Res. 2013, 15, 1-7.

33. M. Olbrich, E. Rebollar, J. Heitz, I. Frischauf and C. Romanin Appl. Phys. Lett. 2008, 92, 013901013903.

34. S. Nie and S. R. Emory Science 1997, 275, 1102-1106.

35. L. Polavarapu and L. M. Liz-Marzan Phys. Chem. Chem. Phys. 2013, 15, 5288-5300.

36. F. C. Cabrera, P. H. B. Aoki, R. F. Aroca, C. J. L. Constantino, D. S. dos Santos and A. E. Job J. Raman Spectrosc. 2012, 43, 474-477.

37. G. Lu, H. Li and H. Zhang Chem. Comm. 2011, 47, 8560-8562.

38. T. Hasell, L. Lagonigro, A. C. Peacock, S. Yoda, P. D. Brown, P. J. A. Sazio and S. M. Howdle Adv. Func. Mater. 2008, 18, 1265-1271.

39. M. Geissler, K. Li, B. Cui, L. Clime and T. Veres J. Phys. Chem. C 2009, 113, 17296-17300.

40. A. Szymczyk Eur. Polym. J. 2009, 45, 2653-2664.

41. E. Rebollar, S. Pérez, J. J. Hernández, I. Martín-Fabiani, D. R. Rueda, T. A. Ezquerra and M. Castillejo Langmuir 2011, 27, 5596-5606.

42. I. Martín-Fabiani, E. Rebollar, S. Pérez, D. R. Rueda, M. C. García-Gutiérrez, A. Szymczyk, Z. Roslaniec, M. Castillejo and T. A. Ezquerra Langmuir 2012, 28, 7938-7945.

43. E. Rebollar, J. R. Vazquez de Aldana, I. Martin-Fabiani, M. Hernandez, D. R. Rueda, T. A. Ezquerra, C. Domingo, P. Moreno and M. Castillejo Phys. Chem. Chem. Phys. 2013, 15, 11287-11298.

44. M. Walczak, M. Oujja, J. Marco, M. Sanz and M. Castillejo Appl. Phys. A 2008, 93, 735-740.

45. D. Bäuerle Laser processing and chemistry; Springer-Verlag: Berlin, 2000.

46. T. H. Joo, M. S. Kim and K. Kim J. Raman Spectrosc. 1987, 18, 57-60.

47. G. Xue, M. Ma, J. Zhang, Y. Lu and K. T. Carron J. Colloid Interface Sci. 1992, 150, 1-6.

48. P. A. Atanasov, N. N. Nedyalkov, A. O. Dikovska, N. Ru, S. Amoruso, X. Wang, R. Bruzzese, K. Hirano, H. Shimizu, M. Terakawa and M. Obara Journal of Physics: Conference Series 2014, 514, 012024.

49. C. A. Smyth, I. Mirza, J. G. Lunney and E. M. McCabe Appl. Surf. Sci. 2013, 264, 31-35.

50. T. Csizmadia, B. Hopp, T. Smausz, Z. Bengery, J. Kopniczky, I. Hanyecz and G. Szabó Advances in Materials Science and Engineering 2014, 2014, 6.

51. M. Futamata Faraday Discuss. 2006, 132, 85-94. 


\section{Figure captions}

Figure 1. AFM height images $\left(4 \times 4 \mu \mathrm{m}^{2}\right)$ of PTT irradiated at the conditions listed in Table 1. (a) and (b) correspond to substrates before and after gold-coating respectively. The upper labels indicate the laser irradiation conditions. The height profile along a $4 \mu \mathrm{m}$ horizontal line is shown below every image.

Figure 2. SERS spectra of BT at a concentration of $10^{-4} \mathrm{M}$ on gold-coated non-nanostructured and nanostructured PTT substrates prepared using the laser irradiation conditions listed in Table 1. The inset shows the spectra of BT in liquid (as supplied, with a purity of $98 \%$ ) in the region of interest. Bands at 917 and $1092 \mathrm{~cm}^{-1}$ are indicated with $\left({ }^{*}\right)$ and $\left(^{* *}\right)$ respectively.

Figure 3. AFM height images $\left(500 \times 500 \mathrm{~nm}^{2}\right)$ of gold coated flat PTT (left) and of the top of one periodic structure formed upon irradiation at $266 \mathrm{~nm}$ and normal incidence and gold coated (right). The height profile along a $250 \mathrm{~nm}$ vertical line (depicted in the image) is shown below every image.

Figure 4. Enhancement factor introduced by the laser induced nanostructures as a function of their period, depth and of the average surface roughness. Open symbols correspond to 
structures prepared by irradiation with circularly polarized light. Lines are shown as visual guides. 
Table 1. Enhancement of the SERS signal introduced by different superficial reliefs (with respect to a gold-coated flat polymer film), period $(L)$, amplitude $(h)$ and roughness $\left(R_{a}\right)$, of PTT films generated under various laser irradiation conditions, i.e. wavelength $(\lambda)$, polarization state, angle of incidence, fluence $(F)$ and number of pulses $(N)$.

\begin{tabular}{lllllllll}
\hline$\lambda$ & Polarization & Angle & $F$ & $N$ & $L$ & $h$ & $R_{a}$ & SERS \\
$(\mathrm{nm})$ & & & $\left(\mathrm{mJ} \cdot \mathrm{cm}^{-2}\right)$ & & $(\mathrm{nm})$ & $(\mathrm{nm})$ & $(\mathrm{nm})$ & enhancement \\
\hline 266 & linear & $0^{\circ}$ & 7 & 1200 & 250 & 50 & 20 & 10 \\
\hline 266 & linear & $45^{\circ}$ & 7 & 1200 & 490 & 110 & 28 & 33 \\
\hline 193 & linear & $0^{\circ}$ & 3 & 600 & 170 & 25 & 6 & 2 \\
\hline 795 & linear & $0^{\circ}$ & 37 & 50000 & 780 & 110 & 30 & 30 \\
\hline 266 & circular & $0^{\circ}$ & 7 & 1200 & $260^{\mathrm{a}}$ & 80 & 23 & 19 \\
\hline
\end{tabular}

${ }^{a}$ this value corresponds to the diameter of the obtained circular structures. 
Figure 1

$266 \mathrm{~nm}, 0^{\circ} \quad 266 \mathrm{~nm}, 45^{\circ} \quad 193 \mathrm{~nm}, 0^{\circ} \quad 795 \mathrm{~nm}, 0^{\circ} \quad 266 \mathrm{~nm}, 0^{\circ}$,

(a) Uncoated

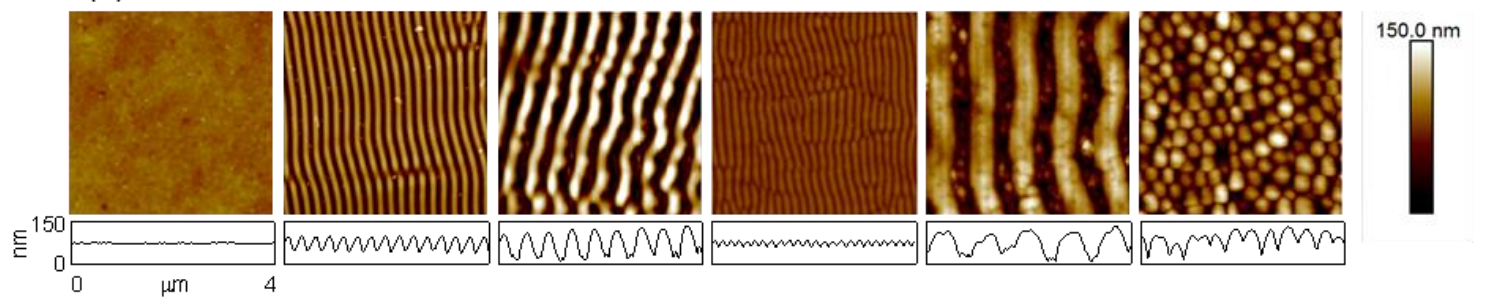

(b) Gold-coated

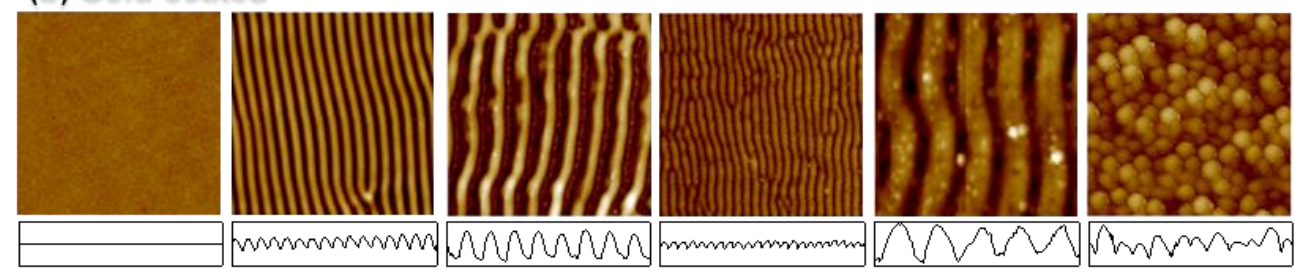


Figure 2

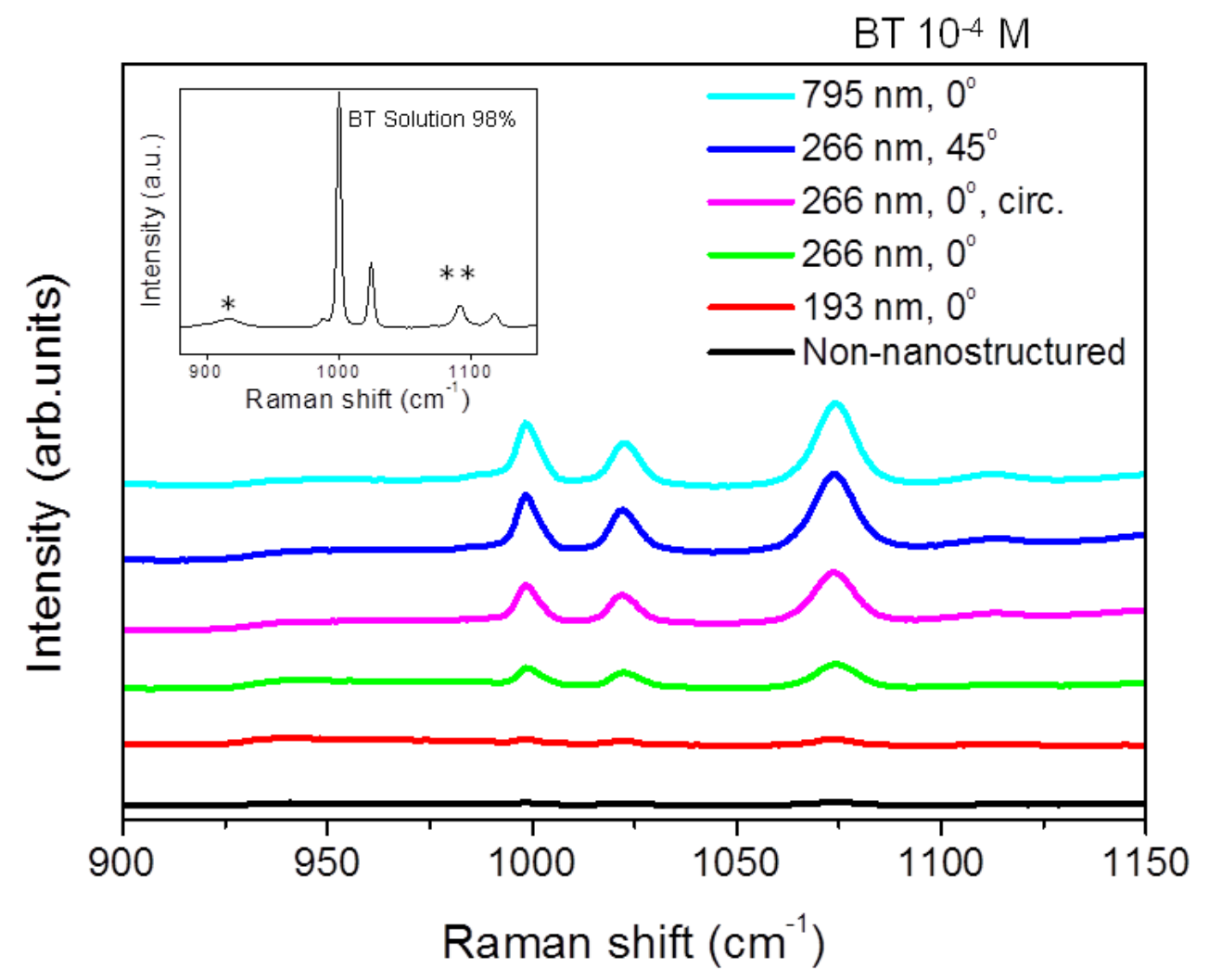


Figure 3
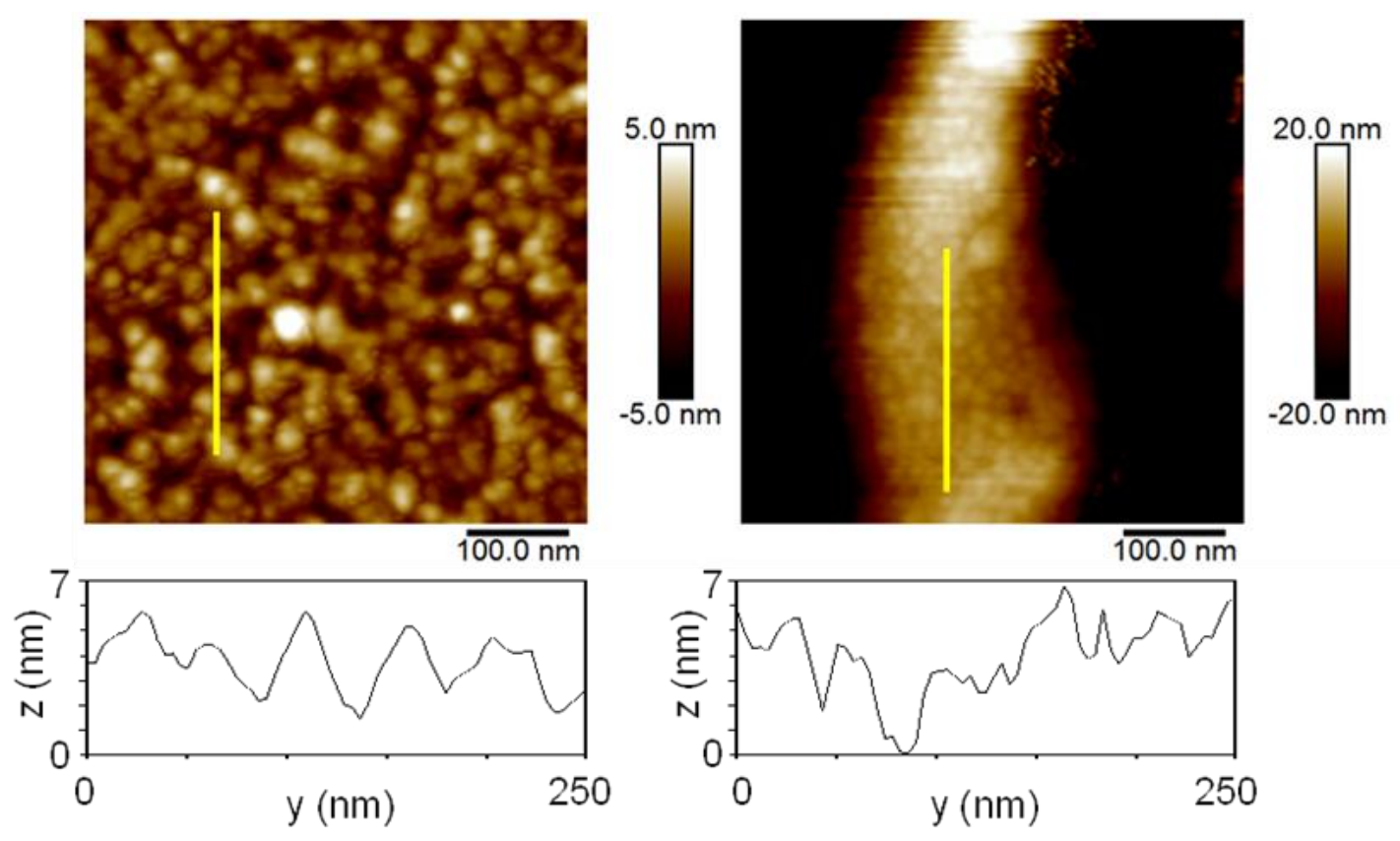
Figure 4

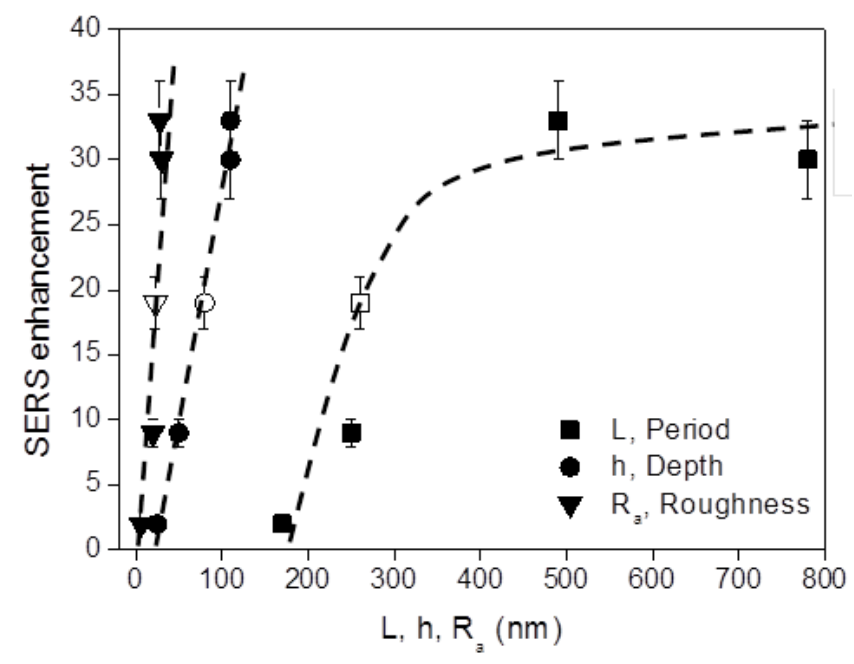

